\title{
A two-year clinical lifestyle intervention program for weight loss in obesity
}

\author{
Karin Andersson', Brita Karlström', Susanne Fredén', \\ Helena Petersson', Margareta Öhrvall ${ }^{2}$ and Björn Zethelius ${ }^{2}$
}

'Department of Public Health and Caring Sciences/Clinical Nutrition and Metabolism, Uppsala University, Uppsala, Sweden; ${ }^{2}$ Department of Public Health and Caring Sciences/Geriatrics, Uppsala University, Uppsala, Sweden

Abstract

Background: In recent randomised prospective studies, lifestyle intervention induced a weight loss of approximately $5 \%$.

Objective: To describe and evaluate a 2-year on-going group intervention program in clinical practice in terms of weight loss and changes in metabolic risk factors, i.e. sagital abdominal diameter (SAD), triglycerides, fasting blood glucose and blood pressure.

Design: The aim of the intervention program was to motivate lifestyle changes concerning food intake and physical activity. The emphasis was on lifestyle modification, followed up at regular visits during 2 years. Subjects evaluated were 100 women with mean BMI $37.6 \mathrm{~kg} / \mathrm{m}^{2}$ and 26 men with mean BMI $36.5 \mathrm{~kg} / \mathrm{m}^{2}$.

Results: One hundred of 151 enrolled women and 26 of 36 men completed the program. Mean weight decreased by $3.8 \mathrm{~kg}$ in women (from 103.5 to $99.7, p<0.001$ ) and $4.4 \mathrm{~kg}$ in men (from 116.5 to 112.1 , $p<0.05)$, respectively. SAD decreased by $5 \%(p=0.001$ in women, $p=0.01$ in men), and triglycerides by $16 \%$ in women $(p=0.01)$ and $24 \%$ in men $(p=0.001)$, however systolic and diastolic blood pressure increased slightly but significantly.

Conclusion: It is possible to perform a clinical lifestyle intervention program for outpatients on an ongoing basis with weight loss, lowered SAD and triglycerides, and a similar or lower dropout rate compared to clinical trials.

Keywords: overweight; weight changes; triglycerides; sagital abdominal diameter; food intake; physical activity

Received: 10 Dec. 2007; Revised: 18 Feb. 2008; Accepted: 20 Feb. 2008

$\mathrm{O}$ besity has been classified as an epidemic according to the WHO (1). In Sweden, the prevalence of obesity has increased over the last decades, and in 2004, according to the WHO definition (1), it was about $12 \%$ (2). Complications of obesity are increasing and there is strong evidence that obesity contributes to a number of metabolic disorders, such as insulin resistance, dyslipidemia, hypertension, hypercoagulability, microalbuminuria, type 2 diabetes mellitus and cardiovascular disease (3-7).

According to the WHO (8), subjects with insulin resistance or type 2 diabetes and two or more of the other risk factors are defined as having the metabolic syndrome. Insulin resistance is considered the key factor of these disorders $(4,5,9,10)$, and there is a need for a simple clinical marker of insulin resistance (11). Central obesity is associated with insulin resistance (7). It has been shown that the sagital abdominal diameter (SAD) is more strongly correlated to insulin sensitivity and the risk factors in the metabolic syndrome compared to BMI, waist girth and waist-hip-ratio (WHR) $(12,13)$. For several years, SAD has been measured at our clinic as a surrogate marker for insulin resistance.

Weight reduction is difficult to obtain and maintain, and the need for better performing treatment is vast (3, 14). Recent studies have shown that despite difficulties in reducing the overweight to normal weight, it is possible to markedly reduce the incidence of diabetes and obtain other beneficial health effects even at a moderate weight loss of approximately 5\% (15-17). In the Diabetes Prevention Study (DPS) and the Diabetes Prevention Program (DPP), through lifestyle changing programs it was possible to reduce the incidence of diabetes by $58 \%$ in subjects with obesity and glucose intolerance $(15,16)$. The lifestyle change also reduced several other cardiovascular risk factors associated with insulin resistance (15). 
Obesity treatment at the Outpatient Clinic for Obesity Care, Uppsala University Hospital, Uppsala, Sweden, addresses obese patients with a BMI $>30 \mathrm{~kg} / \mathrm{m}^{2}$ or a $\mathrm{BMI}>28 \mathrm{~kg} / \mathrm{m}^{2}$ with concomitant metabolic disorders. Since the late 1970s, treatment for overweight has been carried out and developed at the clinic. Currently, about 700 patients are referred to the clinic each year. Among the different individualised treatments available is a 2-year group treatment with the emphasis on lifestyle changes.

The aim of the present work was to describe the 2-year clinical lifestyle intervention program in obesity and to evaluate the changes in body weight and metabolic risk factors, i.e. SAD, triglycerides and blood glucose, and to calculate the drop-out rate over the 2-year programme period.

\section{Material and methods}

\section{Subjects}

The patients were referred to the outpatient Clinic for Obesity Care from general practitioners in Uppsala County and from clinics at the Uppsala University Hospital. A majority of the participants had previously undergone different weight reduction programs, e.g. weight-watchers or other diets, frequently with scant results and often with extra weight gain afterwards. Most patients suffer from complications due to their obesity, e.g. type 2 diabetes, hypertension, dysplipidemia cardiovascular disease or sleep apnoea disorder. Some patients were already taking pharmacological treatment for such diseases. Of the participants, 74\% were women, which reflects the natural inflow of patients to our clinic where women are more prone to seek treatment for obesity.

The participants were referred to the program following a check-up at the outpatient clinic. The criteria for inclusion into the program was: the patient should be $>18$ years of age and have a BMI $>30 \mathrm{~kg} / \mathrm{m}^{2}$ or a BMI $>28$ with concomitant metabolic disorders. Exclusion criteria were inability to follow spoken or written instructions, refusing group treatment or not being able to participate in water gymnastics. Patients with severe heart failure (NYHA III-IV), chronic obstructive pulmonary disease or other conditions that preclude participation in exercise were not included. Such patients were offered other individualised treatment at the clinic. The present work was performed as part of a quality assurance evaluation at the clinic and the on-going lifestyle intervention program.

The patients included in this program evaluation started the lifestyle intervention program during the period between September 1998 and September 1999, and completed the program between 2000 and 2001 .

\section{Design}

The design of the program was conventional dietary advise on food and physical activity combined, focusing on lifestyle changes, by means of group sessions, for 2 weeks of intensive education and follow-up visits during 2 years (for detailed information see Table 1).

\section{The 2-year lifestyle intervention program}

The current lifestyle intervention program started at the clinic in 1995. The program has been developed and improved throughout the years, but the basic design remains the same. The inflow of new participants included in the program is approximately 100 participants a year.

The aim of the program was to motivate the participants to perform lifestyle changes concerning food intake and physical activity. The aim was to achieve a decrease in weight and/or to improve metabolic status. According to the conclusion that beneficial health effects can be

Table 1. Clinical lifestyle intervention program protocol.

\begin{tabular}{|c|c|c|c|c|c|c|c|c|c|c|c|c|c|c|c|}
\hline Visit & $\mathrm{I}$ & 2 & 3 & 4 & 5 & 6 & 7 & 8 & 9 & 10 & II & 12 & 13 & 14 & 15 \\
\hline Week & $\mathrm{I}-2 *$ & 5 & 8 & 14 & 20 & 26 & $32 * *$ & 38 & 44 & 50 & 56 & 68 & 80 & 92 & 104 \\
\hline Weight recording & $X$ & $X$ & $X$ & $X$ & $X$ & $x$ & $\mathrm{X}$ & $\mathrm{X}$ & $\mathrm{X}$ & $\mathrm{X}$ & $\mathrm{X}$ & $X$ & $\mathrm{X}$ & $\mathrm{X}$ & $\mathrm{X}$ \\
\hline SAD recording & $\mathrm{X}$ & & & & & & $\mathrm{X}$ & & & & & & & & $\mathrm{X}$ \\
\hline Biochemical control & $x$ & & & & & & $x$ & & & & & & & & $\mathrm{X}$ \\
\hline Physician & $X$ & & & & & & $X$ & & & & & & & & \\
\hline Dietician & $x$ & $x$ & & $x$ & & $\mathrm{X}^{* * * *}$ & $\mathrm{X}$ & & $x$ & & $\mathrm{X}^{* * * *}$ & & $\mathrm{X}$ & & $x$ \\
\hline Nurse & $x$ & & $x$ & & & & $\mathrm{X}$ & $\mathrm{X}$ & & & & $x$ & & $x$ & \\
\hline Physiotherapist & $x$ & & & & $x$ & & $x$ & & & $x$ & & & & & \\
\hline Almoner & $x$ & & & & & & & & & & & & & & \\
\hline
\end{tabular}

*Two weeks $(80 \mathrm{~h})$ introductory education at the outpatient clinic.

**Two days $(16 \mathrm{~h})$ rehearsal at the outpatient clinic.

****Two hours follow-up visit including dietician supervised cooking. 
attained at a modest level of weight loss, the treatment goal for weight reduction was set to $5-10 \%$ (17). Followup was performed at regular follow-up visits during 2 years (Table 1$)$.

On referral to the program, the patients were given oral and written information at the doctor's appointment. Two weeks before the start of the program, an appointment for repeated information was held for the group. The rules and aims of the program were given and the patients were asked to consider their motivation to participate throughout the entire 2-year program. Informed consent and a written contract were set up and the patients received a start date. Each group consisted of eight to ten members, women and men in separate groups. All data were registered decoded, deleting the individual civic number, thereby making it impossible to identify any given participants data in the dataset. This dataset was used for statistical analyses. The study was performed in accordance with the Helsinki Declaration of 1975, as revised in 1983 and applicable Swedish law.

Lessons - educational program

During the first 2 weeks of the program, the participants received an 80-h introductory lesson at the outpatient clinic. A team of a physician, dieticians, physiotherapists, nurses and almoners worked together according to a specified schedule in order to provide a holistic lifestyle message. The focus was advice on diet and physical activity combined with lifestyle changes. A detailed schedule may be obtained from the author.

The schedule consisted of group sessions given as theory lessons, discussions and practical tasks. Although held in a group, the discussions and tasks focussed on individual behaviour. The purpose was to make the participants aware of their own behaviour and to modify it to a more adequate lifestyle. The new regimen was to be maintained by setting up goals and practising the new habits on an everyday basis.

An individual appointment with the physician in charge was made at the start and end of the initial 2 weeks of the program. Pharmacological treatment was discussed, introduced or altered and prescribed if necessary. The aim was to optimise the metabolic status according to current guidelines, but also to use the results that the patient achieved through changing lifestyle as a pedagogic tool for the maintenance of new lifestyle.

\section{Dietary advice, food content and cooking practice}

The nutritional education was based on the Swedish Dietary Recommendations (SNR) 1997 (18), communicated in theory and practice. The participants were encouraged to change their diet to achieve a balanced intake of fat, between 25 and $30 \%$ of the energy intake with a maximum of $10 \%$ from saturated fat, to increase the intake of fibre, to increase the intake of vegetables and fruits, and to moderate the intake of sugar and alcohol. The balance between the other macronutrients followed the SNR, i.e. $55-60 \%$ of the total energy intake from carbohydrates and 10-15\% from protein. According to SNR, three regular meals and two or three smaller meals, e.g. fruit or bread, were recommended. Without counting calories, the participants were taught to eat in a way that would give them between 1600 and 1800 calories. Theoretical discussions were followed up by dietician supervised practice; each day the participants made their own lunch and dinner to experience the methodology of cooking a balanced meal, to taste it and to feel the satiety after ingestion of a normal sized portion. For these meals, an energy-reduced version of the Swedish plate model (19) was used. The plate model is an easy, pedagogical tool to make a meal balanced, and is based on the principal that about one-third to a half of the plate consists of vegetables and the other part is shared equally between carbohydrate rich food and food rich in protein. This part of the program also included discussions on how to combine the new lifestyle into everyday life, such as planning meals, number of occasions allowing 'extravagance', shopping for food etc.

The participants were asked to perform a 2-day food record before the start of the program. The food record was calculated with a dietician and constituted the basis for individual food counselling.

Physical activity - theory and practice

The central message was to encourage the participants to increase their physical activity independent of their present level. An exercise test was performed at baseline and followed up at week 32. During the initial 2 weeks, each day water gymnastics was a morning activity, and other forms of training sessions took place in the afternoon, e.g. Nordic walking (walking with a walkingstick in each hand) and adapted workout or exercise bicycling. The aim was to enable the participants to discover different forms of activity that they might continue at home on an ongoing individual basis. Further topics discussed by the physiotherapists were injury prevention and the positive effects of physical activity on weight loss, metabolic status and wellbeing.

The follow-up visits

A 14-visit curriculum during 2 years, covering diet, exercise and lifestyle modification followed the introductory education. The aim was to help and support the participants to improve and maintain the new regimen. The pedagogic structure of the follow-up visits was based on different exercises designed as homework, that were individualised and flexible. The role of the supervisor at follow-up visits was altered between the staff members (Table 1). The relationship between the supervisor and the participants was based on collaboration, the super- 
visor leading the motivating discussions and guiding the patients to increase their awareness of their habits and setting up adequate goals for homework. The individual experiences in the groups were brought up for discussion to create an interpatient-based support. The follow-up visits normally lasted $60 \mathrm{~min}$, except two occasions involving cooking. At week 32, the structure of the initial 2 weeks was repeated for 2 days at the outpatient clinic. Weight was checked at every follow-up visit and baseline biochemical and anthropometric measurements at the 32-week rehearsal and at the end of 2 years (Table 1).

\section{Measures}

Anthropometrical and biochemical measurements

Biochemical and anthropometrical status were measured at baseline, at week 32 and at week 104. For patients with high values of blood glucose, lipids or blood pressure, blood samples was recollected during the initial 2 weeks and used as a basis for the discussion with the physician on adjustment of ongoing or possible new pharmacological treatment in addition to lifestyle intervention. Body weight was recorded at each follow-up visit, and was measured to the nearest $0.1 \mathrm{~kg}$ using an electronic scale with subjects wearing light clothing and no shoes. Height was measured to the nearest $0.1 \mathrm{~cm}$ without shoes and BMI $\left(\mathrm{kg} / \mathrm{m}^{2}\right)$ was calculated. SAD $(\mathrm{cm})$ was measured by standardised methods as previously described (12) to the nearest $0.1 \mathrm{~cm}$ after normal expiration while in supine position with legs bent, on a firm examination table. Systolic and diastolic blood pressure (SBP, DBP) was measured in the supine position using a Kifa Ercometer. SBP and DBP were defined as Korotkoff phases I and IV, respectively. Hypertension was defined as SBP $>140$ or DBP $>90 \mathrm{mmHg}$ on 3 visits.

All anthropometrical and biochemical measurements were performed by trained nurses. Blood samples were collected after an overnight fast and centrifuged. Plasma glucose, serum triglycerides (TG), total serum cholesterol and HDL-cholesterol were measured using standardised methods, analysed at the Department of Clinical Chemistry, University Hospital, Uppsala. Diabetes type 2 was defined as a fasting plasma glucose $\geq 7.1 \mathrm{mmol} / 1$ on two occasions.

\section{Data collection and statistical analysis}

Data was collected from patient files and made untraceable for identity. For statistical analysis, software SAS PC 8.0 was used. All statistics were calculated for women and men separately. Descriptive statistics include means and standard deviations. The mean $(\%)$ difference between the laboratory parameters at week 0, week 32 and week 104 were evaluated by Student's paired $t$-test. Differences were considered significant at $p<0.05$. All tests were twotailed.
Table 2. Baseline characteristics of patients completing the lifestyle intervention program.

\begin{tabular}{lcc}
\hline Characteristics & Females & Males \\
\hline Number & 100 & 26 \\
Age (years) & $48 \pm 12$ & $50 \pm 10$ \\
Body weight $(\mathrm{kg})$ & $103.5 \pm 18.1$ & $116.5 \pm 22.5$ \\
Body mass index $\left(\mathrm{kg} / \mathrm{m}^{2}\right)$ & $37.6 \pm 6.2$ & $36.5 \pm 5.3$ \\
Sagital diameter $(\mathrm{cm})$ & $29.0 \pm 2.9$ & $31.5 \pm 3.8$ \\
Total cholesterol $(\mathrm{mmol} / \mathrm{l})$ & $5.6 \pm 1.0$ & $5.5 \pm 1.2$ \\
HDL-cholesterol $(\mathrm{mmol} / \mathrm{l})$ & $1.3 \pm 0.3$ & $1.2 \pm 0.2$ \\
Triglycerides $(\mathrm{mmol} / \mathrm{l})$ & $2.3 \pm 1.2$ & $3.7 \pm 4.5$ \\
Blood glucose $(\mathrm{mmol} / \mathrm{l})$ & $5.7 \pm 1.7$ & $7.0 \pm 3.4$ \\
SBP (mmHg) & $126 \pm 16$ & $131 \pm 17$ \\
DBP (mmHg) & $76 \pm 9$ & $82 \pm 12$ \\
Diabetes type 2 (n (\%)) & $20(20)$ & $9(35)$ \\
Hypertension $(\mathrm{n}(\%))$ & $15(15)$ & $7(27)$ \\
\hline
\end{tabular}

Data presented are arithmetic means \pm standard deviations, number or percent.

\section{Results}

The baseline characteristics of the participants are given in Table 2. The evaluation include more women than men, reflecting the higher proportion of women that are referred to the outpatient clinic.

\section{Dropouts}

One hundred out of 151 women and 26 out of 36 men completed the program. Hence, the dropout rates were 34 and $28 \%$, respectively, in total $33 \%$. To be considered having completed the program, the participants had to attend at least one of the two last follow-up visits (week 90 or week 104). There were no significant differences in age, sex, weight or BMI between those who completed and dropouts.

\section{Weight loss}

During the first 32 weeks, the mean weight decreased among those who completed the course by $5.0 \mathrm{~kg}$ $(6.5 \%)$ in women $(p<0.001)$ and $11.1 \mathrm{~kg}(8.2 \%)$ in men $(p<0.001)$. The initial weight loss was maintained during the first year, but during the second year some of the body weight was regained $(1.2 \mathrm{~kg}$ among women and $6.5 \mathrm{~kg}$ men). However, at 2 years the mean weight was still significantly decreased by almost $4 \%$ in both sexes (Table 3). See Table 3 for changes in BMI. Fig. 1 presents the proportion of patients with a weight loss of $5-10 \%$ and more than $10 \%$ after 2 years.

\section{Obesity-associated risk factors}

After 32 weeks, there was a considerable decrease in triglycerides in both women and men, which was sustained also after 2 years. SAD was decreased by $5 \%$ 
Table 3. Metabolic variables at 32 and 104 weeks in the lifestyle intervention program and percent changes from baseline in program completers.

\begin{tabular}{|c|c|c|c|c|c|c|c|c|}
\hline \multirow[b]{3}{*}{ Variable } & \multicolumn{4}{|c|}{32 weeks } & \multicolumn{4}{|c|}{104 weeks } \\
\hline & \multicolumn{2}{|l|}{ Female } & \multicolumn{2}{|l|}{ Male } & \multicolumn{2}{|c|}{ Female } & \multicolumn{2}{|l|}{ Male } \\
\hline & Mean \pm SD & $\mathrm{n}$ & Mean \pm SD & $\mathrm{n}$ & Mean $\pm S D$ & $\mathrm{n}$ & Mean $\pm S D$ & $\mathrm{n}$ \\
\hline Body weight (kg) & $98.5 \pm 17.9 * * *$ & 80 & $105.4 \pm 21.1 * * *$ & 24 & $99.7 \pm 18.3^{* * * *}$ & 100 & $112.1 \pm 22.7^{*}$ & 26 \\
\hline Change (\%) & $-6.5 \pm 6.0^{* * * *}$ & & $-8.2 \pm 8.1^{* * *}$ & & $-3.5 \pm 7.4 * * *$ & & $-3.7 \pm 7.1^{*}$ & \\
\hline BMI $\left(\mathrm{kg} / \mathrm{m}^{2}\right)$ & $35.7 \pm 6.2^{* * * *}$ & 80 & $33.6 \pm 4.9 * * *$ & 24 & $36.2 \pm 6.3^{* * *}$ & 100 & $34.7 \pm 4.5^{*}$ & 24 \\
\hline Change (\%) & $-6.5 \pm 6.0 * * *$ & & $-8.2 \pm 8.1 * * *$ & & $-3.5 \pm 7.4^{* * *}$ & & $-3.7 \pm 7.1^{*}$ & \\
\hline SAD $(\mathrm{cm})$ & - & & - & & $27.6 \pm 3.5^{* * *}$ & 84 & $30.0 \pm 4.0 * *$ & 18 \\
\hline Change (\%) & - & & - & & $-5.4 \pm 8.0 * * *$ & & $-4.8 \pm 6.8^{* *}$ & \\
\hline Total cholesterol $(\mathrm{mmol} / \mathrm{l})$ & $5.5 \pm 1.1$ & 92 & $5.2 \pm 1.2$ & 21 & $5.5 \pm 1.1$ & 94 & $5.6 \pm 1.0$ & 21 \\
\hline Change (\%) & $-1.3 \pm 13.4$ & & $-0.6 \pm 16.5$ & & $-0.9 \pm 16.0$ & & $1.0 \pm 17.4$ & \\
\hline Triglycerides $(\mathrm{mmol} / \mathrm{l})$ & $1.9 \pm 1.2 * * *$ & 92 & $1.8 \pm 0.9$ & 21 & $1.8 \pm 1.1 * * *$ & 93 & $2.2 \pm 1.0 * * *$ & 21 \\
\hline Change (\%) & $-15.8 \pm 31.3 * * *$ & & $-22.8 \pm 22.4$ & & $-16.1 \pm 33.4^{* * *}$ & & $-24.2 \pm 24.6 * * *$ & \\
\hline Blood glucose $(\mathrm{mmol} / \mathrm{l})$ & $5.2 \pm 1.2^{* * * *}$ & 92 & $5.1 \pm 1.4^{*}$ & 21 & $5.8 \pm 1.9$ & 92 & $6.6 \pm 3.1$ & 20 \\
\hline Change (\%) & $-6.2 \pm 18.0^{* * * *}$ & & $-16.1 \pm 18.0 *$ & & $2.6 \pm 20.2$ & & $-5.9 \pm 20.3$ & \\
\hline Systolic blood pressure & - & & - & & $129 \pm 16$ & 86 & $135 \pm 15$ & 19 \\
\hline Change (\%) & - & & - & & $2.3 \pm 6.2 * *$ & & $3.0 \pm 8.2 *$ & \\
\hline Diastolic blood pressure & - & & - & & $79 \pm 8$ & 86 & $84 \pm 9$ & 19 \\
\hline Change (\%) & - & & - & & $3.9 \pm 9.7 * *$ & & $2.4 \pm 6.5$ & \\
\hline
\end{tabular}

${ }^{*} p<0.05,{ }^{* *} p<0.01,{ }^{* * *} p<0.001, p$-values were determined by Student's paired $t$-test for differences from baseline.

$(p<0.001, p<0.01)$ in both groups at 2 years. Fasting plasma glucose was lowered at 6 months for both women and men, but not after 2 years for women. For men, however, the decrease was not significant after 2 years (for details see Table 3). There were no statistically significant changes in blood cholesterol. Blood pressure was slightly but significantly increased in both men and women at 2 years (Table 3). Diabetes and hypertension

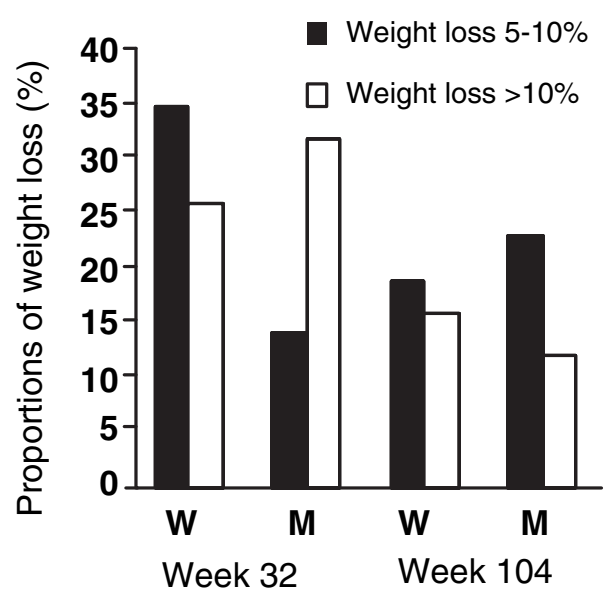

Fig. 1. Frequency of participants obtaining weight loss $\geq 5 \%$. The black bars present percentage of participants losing $\geq 5$ but $<10 \%$. The white bars present percentage of participants losing $>/ 10 \%$. were treated if necessary according to guidelines. However, introduction or withdrawal of the pharmacological treatment of the patients was not registered in the database.

\section{Discussion}

This evaluation showed weight reduction of $-3.8 \mathrm{~kg}$ in women and $-4.4 \mathrm{~kg}$ in men (3.5 and 3.7\%). Therefore, it was possible to obtain results in our continuously used clinical 2-year lifestyle program that are comparable to randomised controlled studies. For instance, in the DPS and DPP studies, the weight loss was -3.5 and $-5.5 \mathrm{~kg}$, respectively after 2 years of treatment $(15,16)$. Lifestyle intervention programs in obesity studies in Sweden have also shown weight reduction of a similar magnitude (20-22). Several studies confirm that lifestyle intervention treatment including diet and physical activity is capable of providing a remarkable improvement in risk factors even at a modest weight loss $(17,21,22)$, and in the long term preventing more severe illness $(15,16,23,24)$

Fasting blood glucose was significantly lowered at 6 months, but not significantly so after 2 years for both sexes. This suggests an acute effect on glucose by the initial weight loss and abolished blood glucose lowering at 2 years may indicate the progressive nature of the diabetic state. However, it should be considered that the average fasting blood glucose was higher in men, even 
above the present cut off limit for type 2 diabetes, but below in women.

In comparison with previous controlled randomised trials $(15,16)$, the limitations in clinical routine care made it impossible to perform an oral glucose-tolerance test every 6 months and comment on type 2 diabetes. However, despite the modest weight loss, the treatment resulted in significant improvements in some important factors. SAD was decreased by $5 \%$ in both women and men, and triglycerides were decreased by as much as $16 \%$ in women and $24 \%$ in men at 2 years. Both measurements are associated with insulin resistance, which is suggested to precede the development of diabetes 2 and cardiovascular disease $(4,5,11,25,28)$. SAD has been shown to correlate to insulin resistance and other signs of disturbed glucose metabolism (12) more strongly than other anthropometric measurements (13). SAD has also shown a strong correlation to mortality in men $(29,30)$. Change in SAD was also related to a change in the metabolic syndrome in the SOS study (22). This highlights that measurement and decrease in SAD is of clinical importance.

Triglycerides were lowered by $20 \%$, whereas total serum cholesterol was not decreased, which indicates that the lowering of TG was induced by weight loss and lowered central obesity and not by the introduction of lipid lowering medication, such as statins. However, the overall aim was to optimise metabolic control. Further, we cannot exclude that a few patients used anti-obesity drugs for a short period of time at the end of the program evaluation period. Orlistat, which was the only antiobesity drug available for prescription at that time, was not subsidised and thus very expensive for the individual patient. Hence, it was most probably not used often by patients included in this evaluation.

Obesity is a chronic condition and calls for life-long treatment (1), which is a demanding task. Although public health care has to deal with the consequences of obesity, the possibilities for obesity treatment are few. The pedagogical structure of our out-patient lifestyle program was to motivate the participants to make stepwise improvements in eating patterns and physical activity, which are unlikely to be maintained (31). The participants were encouraged to keep their new habits in a long term, and learning maintenance strategies was a part of the program. The initial weight loss was maintained during the first year, as long as the follow-up visits were held every 6 weeks, but during the second year a slight body weight regain was observed. This effect has been shown in other obesity treatments $(16,20,21,32)$. An optimal structure and pedagogic model for long term weight stability is not yet defined and further development in this area is needed.

The group plays an important role as social support, which is correlated to weight maintenance (31). Our experience is that group treatment is not only cost cutting and time-saving compared with individual counselling, but often creates a base for recognition and support. Here, the group discussion was used as a pedagogical tool. For instance, it may be good for a participant to get feedback from group members, in addition to feedback from health care professionals. When a group is well functional, friendship and security are created. Our clinical experience is that a well-functioning and intact group throughout the whole program improves results. This, however, has not been formally evaluated. Further, such groups tend to continue to work after the 2-year program without support from the clinic, according to reports from individual patients.

The dropout frequency in our 2-year lifestyle intervention program was $33 \%$, which is comparable to other long-term obesity treatment $(20,21,33)$. For instance, the Gustaf-study had a dropout of 34\% (21), the XENDOSstudy $37 \%$ (20), and the study by Melin et al. of 53\% (33) after 2 years. Dropout rates also differ between studies due to differences in study designs, but are obviously possible to keep at the same level in a clinical setting used in the present study.

In summary, our 2-year lifestyle obesity program for out-patient clinic patients could be considered well performing and the treatment effect was as good as in other lifestyle intervention programs and in randomised controlled studies. Weight loss and reduced central obesity improved metabolic risk factors after 2 years of treatment, making it probable that the conclusions drawn in randomised controlled studies, that type 2 diabetes and related complications may be prevented or postponed $(15,16,23,24)$, also apply to a 2-year lifestyle intervention program such as the program presented here. It would be realistic to implement such a lifestyle intervention program in primary health care. However, further research is needed on the long-term effects.

SAD has been suggested to be the most useful and simple tool to identify insulin resistance and is easily implemented in the clinic. Studies to compare waist circumference measurements and SAD for measuring central obesity in a clinical setting are warranted.

\section{Conflict of interest and funding}

This work was supported by Uppsala University Hospital Fund for Clinical Development.

\section{References}

1. WHO (1997): Obesity: preventing and managing the global epidemic. WHO Technical Report Series, No. 894. Geneva: World Health Organisation.

2. Persson U, Ödegard K. Indirekta kostnader till följd av sjukdomar relaterade till övervikt och fetma. IHE, e-report 2003:5, pp 1-20, www.ihe.se. 
3. SBU. Obesity a grave epidemic. The Swedish council on technology assessment in health care (2002) report number 160, pp 401-59, www.sbu.se. ISBN: 91-87890-78-X.

4. Reaven G. Metabolic syndrome: pathophysiology and implications for management of cardiovascular disease. Circulation 2002; 106: 280-8.

5. Ginsberg HN. Insulin resistance and cardiovascular disease. J Clin Invest 2002; 106: 453-8.

6. Isomaa B, Almgren P, Tuomi T, Forsen B, Lathi K, Nissen M, et al. Cardiovascular morbidity and mortality associated with the metabolic syndrome. Diabetes Care 2001; 24: 683-9.

7. Depres JP. Abdominal obesity as important component of insulin-resistance syndrome. Nutrition 1993; 9: 452-9.

8. Alberti KG, Zimmet PZ. Definition, diagnosis and classification of diabetes mellitus and its complications. Part 1: diagnosis and classification of diabetes mellitus provisional report of a WHO consultation. Diabetes Med 1998; 15: 539-53.

9. DeFronzo RA, Bonadonna RC, Ferrannini E. Pathogenesis of NIDDM. A balanced overview. Diabetes Care 1992; 15: 318-68.

10. Zavaroni I, Bonini L, Gasparani P, Barilli AL, Zuccarelli A, Dall'Aglio E, et al. Hyperinsulinemia in a normal population as a predictor of non-insulin-dependent diabetes mellitus, hypertension, and coronary heart disease: The Barilla factory revisited. Metabolism 1999; 48: 989-94.

11. McLaughlin TL, Reaven GM. Beyond type 2 diabetes: the need for a clinical useful way to identify insulin resistance (Editorial). Am J Med 2003; 114: 501-2.

12. Riserius U, Ärnlöv J, Brismar K, Zethelius B, Berglund L, Vessby B. Sagital abdominal diameter is a strong anthropometric marker of insulin resistance and hyperproinsulinemia in obese men. Diabetes Care 2004; 27: 2042-6.

13. Öhrvall M, Berglund L, Vessby B. Sagital abdominal diameter compared with other anthropometric measurements in relation to cardiovascular risk. Int J Obes 2000; 24: 497-501.

14. Zimmet P, Alberti KG, Shaw J. Global and societal implications of the diabetes epidemic. Nature 2001; 414: 782-7.

15. Toumiletho J, Lindström J, Eriksson J, Valle T, Hämiläinen H, Ilanne-Parrika $\mathrm{P}$, et al. Prevention of type 2 diabetes mellitus by changes in lifestyle among subject with impaired glucose tolerance. N Engl J Med 2001; 344: 1343-50.

16. Knowler WC, Barret-Connor E, Fowler SE, Hamman RF, Lachin JM, Walker EA, et al. Reduction in the incidence of type 2 diabetes with lifestyle intervention and metformin. N Engl J Med 2002; 346: 393-403.

17. Goldstein DJ. Beneficial health effects of modest weight loss. Int J Obes 1992; 16: 397-415.

18. Svenska Livsmedelsverket (Swedish National Food Administration). Svenska näringsrekommendationer (Swedish dietary recommendations) [In Swedish]. Vår föda 1997; 2: 8-14.

19. Karlström B, Vessby B, Eliasson M. Diet - a balanced approach, 1989. Diabetes 1988. Proceedings of the 13th Congress of the International Diabetes Federation, Sydney, 20-25 November 1988.

20. Sjöström L, Rissanen A, Andersen T, Boldrin M, Golay A, Koppeschaar HPF, et al. Randomised placebo-controlled trial of orlistat for weight loss and prevention of weight regain in obese patients. Lancet 1998; 352: 167-72.

21. Andersson I, Rössner S. Weight development, dropout pattern and changes in obesity-related risk factors after two years of treatment of obese men. Int J Obes 1997; 21: 211-6.

22. Sjöström CD, Lissner L, Sjöström L. Relationships between changes in body composition and changes in cardiovascular risk factors: the SOS Intervention Study. Swedish Obese Subjects. Obes Res 1997; 5: 119-30.

23. Pan XR, Li GW, Hu YH, Wang JX, Yang WY, An ZX, et al. Effects of diet and exercise in preventing NIDDM in people with impaired glucose tolerance. Diabetes Care 1997; 20: 537-44.

24. Eriksson KF, Lindgarde F. Prevention of type 2 (NID) diabetes mellitus by diet and physical exercise: the 6-year Malmö feasibility study. Diabetologia 1991; 34: 891-8.

25. The DECODE Study Group. Glucose tolerance and mortality: comparison of WHO and American Diabetes Association diagnostic criteria. Lancet 1999; 354: 617-21.

26. McLaughlin T, Abbasi F, Cheal K, Chu J, Lamendola C, Reaven G. Use of metabolic markers to identify overweight individuals who are insulin resistant. Ann Intern Med 2003; 139: 802-9.

27. Berglund L, Lithell H. Prediction models for insulin resistance. Blood Press 1996; 5: 274-7.

28. Hokansson JE, Austin MA. Plasma triglyceride level is a risk factor for cardiovascular disease independent of HDL cholesterol level: a meta analysis of population based prospective studies. J Cardiovasc Risk 1996; 2: 213-9.

29. Kahn HS, Simoes EJ, Koponen R. The abdominal diameter index and sudden coronary death in men. Am J Cardiol 1996; 78: $961-4$.

30. Seidel JC, Andres R, Sorkin JD, Muller DC. The sagital waist diameter and mortality in men: the Baltimore Longitudinal Study on Ageing. Int J Obes 1994; 18: 61-7.

31. Foreyt JP, Goodrick GK. Factors common to successful therapy for obese patients. Med Sci Sports Exerc 1991; 23: 292-7.

32. Hansen D, Astrup A, Toubro S, Finer N, Kopelman P, Hilsted J, et al.; for the STORM Study Group M. Predictors of weight loss and maintenance during 2 years of treatment of subutramine in obesity. Results from the European multi-centre STORM-trial. Int J Obes Relat Metab Disord 2001; 25: 496-501.

33. Melin I, Reynisdottir, Berglund L, Zamfir M, Karlström B. Motivating clinical treatment of obesity, methods, education, supervision and outcome. Thesis, paper No. 4, Karolinska University Hospital Huddinge, Stockholm, 2004.

\section{Brita Karlström}

Dietistavdelningen, Överviktsenheten, Samariterhemmets sjukhus

75। 85 Uppsala, Sweden

Phone: +46-18-6117984

Fax: +46-18-6117976

E-mail: brita.karlstrom@pubcare.uu.se 